\title{
Überlegungen zur Nutzung schematherapeutischer Konzepte in der Selbsterfahrung bei der Ausbildung von Verhaltenstherapeuten
}

\author{
Gitta A. Jacob \\ Praxis für Psychotherapie, Au, Deutschland
}

\author{
Schlüsselwörter \\ Aus- und Weiterbildung · Selbsterfahrung . \\ Emotionsregulation - Schematherapie
}

\section{Zusammenfassung}

In den vergangenen Jahren werden in die Verhaltenstherapie (VT) zunehmend emotionsaktivierende und -verändernde Interventionen zusätzlich zu Expositionsverfahren integriert. Dabei liegen bisher kaum Konzepte zum gezielten Einsatz solcher Interventionen vor. Ein möglicher Rahmen für den Einsatz emotionaler Arbeit in der VT ist das Schema-Modus-Konzept nach Jeffrey Young bzw. Arnoud Arntz. Eine zunehmende Zahl von Studien zeigt sowohl gute Effekte für emotionsaktivierende Interventionen als auch für die Wirksamkeit der Schematherapie bei verschiedenen Persönlichkeitsstörungen.

Selbsterfahrung ist in Deutschland integraler Bestandteil der VT-Ausbildung. Sie dient gleichermaßen dem Verfolgen praxis- wie personbezogener Ziele. In dieser Arbeit werden einige Überlegungen dazu dargestellt, wie der Schema-Modus-Ansatz in der verhaltenstherapeutischen Selbsterfahrung eingesetzt werden kann. Damit kann praxisbezogen - der systematische Einsatz emotionaler Methoden erfahren und erlernt werden; personbezogen können dysfunktionale Muster von Therapeuten, etwa übermäßige Verantwortungsübernahme oder die Vermeidung von Konfrontation, in ihrem biografischen Bezug geklärt und verändert werden. Eine empirische Überprüfung dieses Ansatzes steht allerdings noch aus.

\author{
Keywords \\ Psychotherapy training - Personal Therapy . \\ Emotion regulation . Schema therapy
}

\section{Summary}

The Schema Therapy Approach in Personal Therapy for CBT Trainees

Emotion activating and modifying interventions are, in addition to expositional techniques, increasingly used by cognitive behavioural therapy (CBT) therapists in the last few years. However, systematic concepts of how to use these interventions are lacking so far. The schema mode model as proposed by Jeffrey Young or Arnoud Arntz is a possible frame for using emotion focussed interventions in CBT treatments. An increasing number of trials show both good effects for emotion focussed methods and high effectiveness of schema therapy in different personality disorders.

In Germany, personal therapy is an integral part of CBT training. Goals are both personal and practical learning. This manuscript describes some considerations how the schema mode model can be used in CBT personal therapy. Therapists can learn in practice how to use emotional methods systematically; with regard to personal goals, dysfunctional patterns of therapist behaviours, such as excessive feelings of responsibility or avoidance of confronting interventions, can be understood in their biographical context and changed. An empirical test of this model would be desirable.

\section{KARGER \\ Fax +497614520714 \\ Information@Karger.de}

www.karger.com

\section{(C) 2011 S. Karger GmbH, Freiburg}

1016-6262/11/0213-0188\$38.00/0

Accessible online at:

www.karger.com/ver
Dr. Gitta A. Jacob

Praxis für Psychotherapie

Im Merzental 38, $79280 \mathrm{Au}$, Deutschland

Tel. +49 761401 10-14, Fax -13

praxis@gitta-jacob.de 


\section{Einführung}

In der kognitiven Verhaltenstherapie (KVT) wird traditionell nicht nur mit kognitiven und behavioralen Methoden gearbeitet, sondern auch mit Interventionen, die das problematische emotionale Erleben des Patienten direkt thematisieren, aktualisieren und verändern, etwa im Rahmen einer Expositionstherapie bei der Behandlung von Angst- und Zwangsstörungen. Darüber hinaus setzten sich in den vergangenen Jahren mit Stuhldialogen, Ansätzen der «Teile-Arbeit» oder dem sogenannten imaginativen Überschreiben (imagery reprocessing and rescripting; ImRS) [Holmes et al., 2007] immer stärker auch emotionsfokussierende Methoden in der Verhaltenstherapie (VT) durch, mit denen insbesondere auch Affekte wie Scham, Angst vor Bedrohung, Selbsthass, Schuld oder Wut aktualisiert und verändert werden können. Der Einsatz solcher Methoden zusätzlich zu Expositionsverfahren lässt sich damit begründen, dass sie auch bei Persönlichkeitsstörungen eingesetzt werden können [Weertman und Arntz, 2007] und dass damit auch andere problematische Emotionen als Angst verändert werden können [Arntz et al., 2007]. Zudem verringert sich die Wahrscheinlichkeit des Wiederauftretens konditionierter Angst beim Einsatz vom ImRS im Vergleich zu reiner Expositionstherapie [Hagenaars und Arntz, persönliche Mitteilung].

Der Einsatz solcher Konzepte erfolgt aktuell häufig in Abhängigkeit von den Präferenzen und Erfahrungen der jeweiligen Therapeuten, explizite Anleitungen zum gezielten, konzeptuell fundierten Einsatz liegen bisher kaum vor. Der Schema-Modus-Ansatz sensu Young et al. [2005] bzw. Jacob und Arntz [2011] kann nach den bisherigen klinischen Erfahrungen als Modell betrachtet werden, mit dem gezielt der Einsatz und die jeweiligen Ziele solcher emotionsorientierter Techniken geplant werden können.

Nach dem Schema-Modus-Ansatz werden problematische Emotionen und Verhaltensmuster, die als dauerhafte Muster bei einem Menschen auftreten, in Abhängigkeit von ihrer emotionalen Qualität folgenden «Anteilen» bzw. SchemaModi zugeordnet:

- Intensive negative Gefühle wie Traurigkeit, Angst, Einsamkeit, Hilflosigkeit, Verzweiflung, bzw. auch Wut, Ärger oder Zorn werden den «Kindmodi» zugeordnet, wobei vulnerable (einsame, traurige, verängstigte) und wütend/ impulsive Kindmodi unterschieden werden.

- Druck auf sich selbst oder Selbsthass werden als «dysfunktionale (strafende oder fordernde) Elternmodi» bezeichnet. Dies entspricht dem psychodynamischen Konzept der Täterintrojekte, wobei nicht zwingend immer die Eltern für die Entwicklung dieser Modi Pate standen. Im Gegenteil berichten Patienten häufig von traumatischen Erlebnissen mit anderen Bezugspersonen, insbesondere auch mit Peer Groups.

- Als «Bewältigungsmodi» werden Verhaltens- und Erlebensweisen bezeichnet, mit denen Patienten die schmerz-

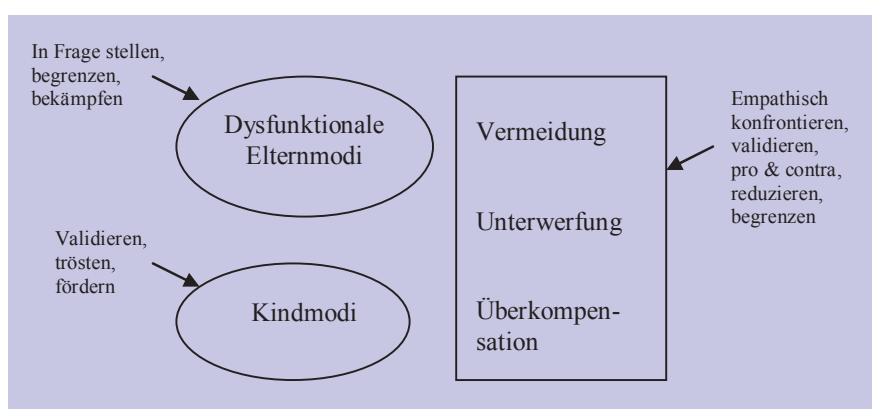

Abb. 1. Interventionsplanung mit dem Schema-Modus-Konzept.

haften Gefühle, die aus den Kind- und Elternmodi resultieren, vermeiden (etwa durch Alkoholkonsum, Vermeidung von Kontakt, sozialem Rückzug, usw.) oder überkompensieren (etwa mit Dominanz, aggressivem Verhalten, usw.). Es besteht eine konzeptuelle Nähe zum psychodynamischen Konzept der Abwehr.

In der Therapie nach diesem Konzept werden zunächst das Problemverhalten und die Symptome eines Patienten in einem modusspezifischen Fallkonzept zusammengefasst. Dabei wird davon ausgegangen, dass der Entwicklung psychischer Störungen in der Regel Vulnerablitätsfaktoren zugrunde liegen. Darüber hinaus besitzt die Ableitung dieser Muster aus der Biografie des Patienten einen hohen Stellenwert. Dies trägt der steigenden Flut an empirischen Belegen Rechnung, nach denen psychische Störungen im Erwachsenenalter mit traumatischen Erfahrungen in der Kindheit assoziiert sind [Lobbestael et al., 2010] und dass ImRS bei traumatischen Kindheitserinnerungen für Patienten mit Persönlichkeitsstörungen hilfreich ist [Weertman und Arntz, 2007].

Die Behandlung folgt dann diesem Fallkonzept, wobei jeder Moduskategorie jeweils bestimmte Behandlungsziele und -strategien zugeordnet werden. Abbildung 1 gibt eine Übersicht über die Modi und die damit zusammenhängenden Behandlungsziele. Der Einsatz des Modells in der Arbeit mit Patienten im Allgemeinen ist bei Jacob und Arntz [2011] genauer beschrieben.

Schematherapie hat sich bisher in mehreren Studien für die Behandlung der Borderline-Persönlichkeitsstörung als wirksam erwiesen [Farrell et al., 2009; Giesen-Bloo et al., 2006; Nadort et al., 2009]. Erste ermutigende Daten zur Wirksamkeit bei Patienten mit Cluster-C-Persönlichkeitsstörung und bei forensischen Patienten werden aktuell berichtet [Arntz, 2010; Bernstein, 2010], für verschiedene Störungsgruppen und Settings werden momentan Konzepte entwickelt und getestet [Roediger und Jacob, 2010].

In dieser Arbeit soll dargestellt werden, wie dieses Modell speziell auch in der (patientenorientierten) Selbsterfahrung eingesetzt werden kann. In Deutschland ist Selbsterfahrung ein obligatorischer Teil der VT-Ausbildung, der einerseits praxisbezogene Ziele in dem Sinne verfolgt, dass Therapeuten in der eigenen Erfahrung und am Modell therapeutische 
Techniken und Prozesse kennen lernen, Handlungskompetenzen aufbauen und ihren eigenen therapeutischen Stil reflektieren sollen. Zusätzlich werden durch Selbsterfahrung personbezogene Ziele in dem Sinne verfolgt, dass angehende Therapeuten dadurch eigene problematische Inter-aktionen und persönliche Belastungen reduzieren und persönliche Ressourcen aufbauen sollen [Laireiter, 2000a].

Zur Gestaltung der Selbsterfahrung liegen verschiedene und teilweise sehr unterschiedliche Ansätze vor [Laireiter, 2000b; Lieb, 1998]. Diese erfüllen übereinstimmend die Prinzipien der theoretischen Fundiertheit in den Grundlagen der VT, der Zielorientierung, der Konzeptgebundenheit und der grundsätzlichen Orientierung an Evaluation und Weiterentwicklung [Laireiter, 2000a]. In der VT wird dabei die Frage kontrovers diskutiert, inwieweit in der Selbsterfahrung im Rahmen der VT-Ausbildung ausschließlich die zentralen Interventionen der VT eingesetzt werden sollten, mit dem primären Ziel, dass Ausbildungskandidaten eben diese Interventionen «am eigenen Leib kennen lernen». Dem wird entgegengesetzt, dass fraglich ist, ob die Anwendung von Methoden zur Beseitigung von Störungen bei Nicht-Vorliegen dieser Störungen sinnvoll ist und dass die Selbsterfahrung in der VTAusbildung auch dem Zweck dienen sollte, individuelle Anliegen und Muster der angehenden Therapeuten zu bearbeiten, selbst wenn diese keinen Symptomwert haben, der den Einsatz klassischer VT-Methoden nahelegt [für eine aktuelle Zusammenfassung dieser Diskussion siehe Rief et al., 2009].

In der vorliegenden Arbeit wird der letztere Standpunkt eingenommen. Im Folgenden wird erläutert, wie der SchemaModus-Ansatz sowohl mit praxis- als auch personenbezogenen Zielen in der Selbsterfahrung eingesetzt werden kann. Der Schwerpunkt liegt auf patientenbezogener Selbsterfahrung, allerdings ist der Übergang sowohl zur Supervision als auch zur generellen Arbeit an persönlichen Mustern fließend.

\section{Dysfunktionale Muster von Therapeuten}

Aus der Burnout-Forschung ist bekannt, dass die Beschäftigung mit psychisch gestörten Patienten zu erheblichen Einbußen im Wohlbefinden der Therapeuten führen kann, mit Symptomen wie körperlicher und geistiger Erschöpfung, Gereiztheit, zynischem Verhalten gegenüber Patienten, usw. Dies ist grundsätzlich für therapeutische Anfänger, die noch sehr enthusiastisch sind und in Therapien ein sehr hohes Maß an Verantwortung übernehmen, in besonderem Maße zutreffend. Das Erleben von Hilflosigkeit, Versagen und Erschöpfung kann die Folge sein [Frank, 2000]. In Ausbildung und Supervision werden immer wieder typische Schwierigkeiten deutlich [Jacob et al., 2009]. Häufig haben (gerade wenig erfahrene) Therapeuten zu hohe Ansprüche an den Erfolg der Therapie, sie erwarten ein Ausmaß an Veränderung, das mit den gegebenen Ressourcen des Patienten gar nicht erreicht werden kann. Dafür engagieren sie sich in extremem Maß, oft

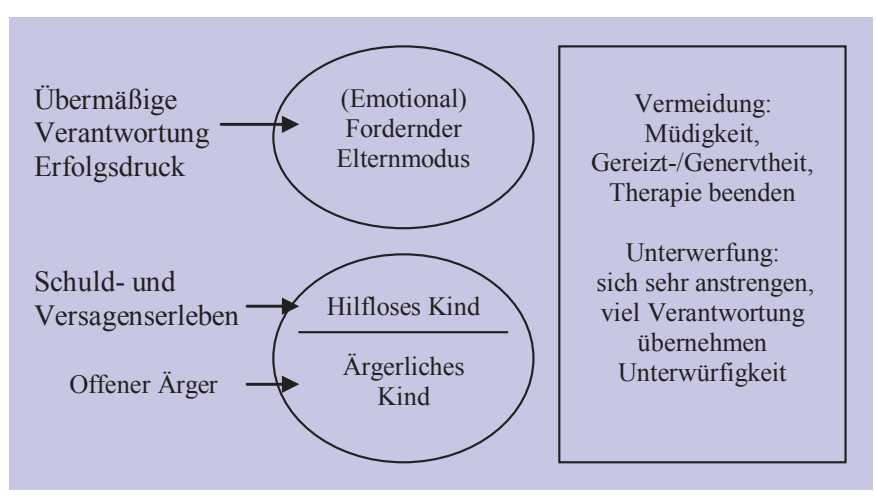

Abb. 2. Typisches Modusmodell von Therapeuten.

unter hohem Druck und mit dem Gefühl, stark für den Fortschritt des Patienten verantwortlich zu sein. Wenn sich erwünschte Erfolge nicht einstellen, treten Unsicherheits- und Versagenserleben auf. Zudem fällt es vielen Therapeuten sehr schwer, Patienten, die dauerhaft dysfunktionale Interaktionsmuster zeigen und so die Therapie behindern (z.B. unablässig klagen, sich nicht festlegen, von emotional belastenden Themen ablenken, die Therapie abwerten), mit diesen Mustern zu konfrontieren.

Gerade im Verlauf wenig erfolgreicher Therapien lassen sich unterschiedliche Bewältigungsmechanismen bei Therapeuten beobachten. Viele Therapeuten sind eher «zu sehr vorgelehnt» und in der Therapie aktiver als der Patient. Andere berichten davon, dass sie in Behandlungen ermüden, gegebenenfalls in den Sitzungen sogar fast einschlafen. Auch Ärger kann auftreten - im Kontakt mit dem Patienten häufig eher in Form von Gereiztheit, in geschützteren Situationen, wie etwa bei Intervisionstreffen, unter Umständen auch mit offener Wut. Exploriert man die Hintergründe solcher Muster in der Biografie des Therapeuten, so findet sich erfahrungsgemäß häufig das Thema einer emotional fordernden Bezugsperson, für deren Wohlergehen sich der Therapeut als Kind verantwortlich gefühlt hat (siehe Fallbeispiel 1). Schematherapeutisch betrachtet weisen Therapeuten, insbesondere wenn ihre Biografie der der Kollegin aus dem Fallbeispiel 1 ähnelt, häufig die Schemata der emotionalen Deprivation, der Unterwerfung und/oder der Selbstaufopferung auf. Im Modusmodell lässt sich dies als emotional fordernder Elternmodus konzeptualisieren, wobei «emotional fordernd» in diesem Zusammenhang bedeutet, dass sich die Forderungen an sich selbst insbesondere auf das Wohlergehen anderer beziehen: Andere, für die ich zuständig bin, dürfen sich nicht unglücklich fühlen, nicht weinen, von mir keine unerfreulichen Dinge gesagt bekommen usw. Im vulnerablen Kindmodus fühlen sich die Betroffenen in entsprechenden Situationen oft hilflos oder schuldig oder sie werden (oft erst nach längerer Zeit) ärgerlich. Die Bewältigungsformen sind meist unterwerfend oder vermeidend, selten auch überkompensatorisch, z.B. wenn Patienten Vorwürfe gemacht werden (Abb. 2). 


\section{Einsatz des Schema-Modus-Konzeptes in Supervision und Selbsterfahrung}

In der (patientenorientierten) Selbsterfahrung kann dieses Modell nach den bisherigen Erfahrungen analog zum therapeutischen Vorgehen eingesetzt werden. Das bedeutet, dass bei ungünstigen Verhaltensweisen des Therapeuten in der Patientenarbeit zunächst exploriert wird, ob es sich dabei um ein dauerhaftes Muster oder eher um ein singuläres Problem handelt. Sofern es sich um ein Muster zu handeln scheint, wird der biografische Bezug geklärt und die beteiligten Emotionen durch emotionsfokussierende Verfahren so umstrukturiert, dass der Therapeut in der Arbeitssituation freier und funktionaler agieren kann. Dabei lassen sich grundsätzlich die gleichen Interventionen, etwa Imagination zur Klärung des biografischen Hintergrundes und/oder zum emotionalen Überschreiben schwieriger oder traumatischer Erinnerungen, Stuhldialoge zur Reduktion des fordernden Elternmodus sowie Imaginationen oder Rollenspiele zur Implementierung neuer Muster einsetzen.

\section{Falldarstellungen}

\section{Fallbeispiel 1}

Fallbeispiel biografischer Hintergrund eines emotional fordernden Elternmodus: Luise M., eine VT-Ausbildungskandidatin, berichtet von einer Patientin, die sie als sehr schwierig erlebt. Es handele sich um eine ältere Frau, die unablässig klage und jammere, insbesondere über Schmerzen, aber auch über die Ungerechtigkeit ihres Schicksals, die Verfehlungen anderer Menschen usw. Sie fühle sich unter größtem Druck, der Patientin zu helfen und habe Schuldgefühle, wenn es der Patientin nicht besser gehe. Alle ihre therapeutischen Vorschläge und Interventionen «prallten jedoch regelrecht ab». Diese Patientin ginge ihr auch nach der Arbeit kaum aus dem Kopf. Die Supervisorin leitet die Kandidatin zu einer Imaginationsübung an: Ausgehend vom Überforderungsund Schulderleben in der aktuellen Situation mit der Patientin, versetzt sich die Kandidatin in ihre Kindheit. Sie «landet» in einer Szene, in der sie als etwa 10-jähriges Kind mit ihrer Mutter allein ist. Die Mutter weint und klagt über Schmerzen und über ihre Eheschwierigkeiten, die kleine Luise fühlt sich für das Wohlergehen der Mutter verantwortlich und versucht sie zu trösten, dabei fühlt sie sich allein und überfordert.

\section{Fallbeispiel 2}

Nina F., eine 34-jährige Verhaltenstherapeutin mit mehreren Jahren Berufserfahrung, berichtet in der Supervision, dass sie narzisstischen Patienten keine angemessenen Grenzen setzen könne. «Ich weiß, dass das wichtig wäre, aber wenn ich nur darüber nachdenke, wie ich eine Grenze formulieren könnte, bekomme ich schon Panik. Letztlich lasse ich immer wieder den Patienten die Stunde dominieren. Manchmal führt das dazu, dass ich Themen, die eigentlich sehr wichtig wären, aus einer diffusen Angst vor Bedrohung heraus nicht anspreche. Auch im Privatleben lasse ich mich von narzisstischen Personen leichter einschüchtern, als mir lieb ist und unterwerfe mich ihren Vorstellungen häufig. Ich weiß wirklich nicht, warum es mir so schwer fällt, meine Positionen besser zu vertreten.»

Um dieses Phänomen genauer zu verstehen und zu explorieren, wird ein Stuhldialog durchgeführt. Die Gefühle von Panik und Bedrohtheit werden dem Stuhl der «ängstlichen Nina» zugeordnet. Die vermeidenden Strategien (andere gewähren und dominieren lassen) werden einem ver- meidenden Beschützer-Modus zugeordnet. Das Wissen darum, dass es gerade bei Patienten mit Persönlichkeitsstörung wichtig sein kann, deren dysfunktionale interpersonelle Muster direkt anzusprechen, wird der «gesunden Erwachsenen» zugeordnet. Die Kollegin nimmt zunächst auf dem Stuhl der «gesunden Erwachsenen» Platz und erklärt, warum sie einen aktuell von ihr behandelten narzisstischen Patienten eigentlich mit einem schwierigen Thema konfrontieren sollte. Dabei steigt die berichtete Angst in ihr hoch. Sie wechselt daher auf dem Stuhl der «ängstlichen Nina», hier wird die Angst exploriert. Die Angst bezieht sich darauf, verprügelt, verfolgt und «fertig gemacht» zu werden. Durch die Exploration steigt zunächst die Angst, nach nur kurzer Zeit jedoch erkennt die Kollegin durch diese Emotionsinduktion den biografischen Hintergrund ihres Problems. Im Alter von 14 Jahren sei sie mehrere Monate von einem älteren Mitschüler verfolgt worden, weil sie ein Fehlverhalten von ihm an die Schulleitung gemeldet hatte; dies habe aufgrund weiterer bereits vorliegender Fehlverhaltensweisen zum Schulausschluss geführt. Der Mitschüler hätte daraufhin gedroht «sie fertig zu machen». Da sie Angst gehabt habe, als «Petze» dazustehen, habe sie sich niemandem anvertraut, aber über Monate in größter Angst gelebt, die Schule immer in Begleitung oder durch den Hinterausgang betreten und verlassen, usw.

Nachdem nun der traumatische Hintergrund der aktuellen Problematik deutlich geworden ist, wird eine Imaginationsübung mit Rescripting durchgeführt [sensu Holmes et al., 2007]. Die Kollegin imaginiert sich in eine Situation als Schülerin, in der ihr Verfolger im Schulhaus auftaucht und auf sie zukommt. Das Rescripting besteht darin, ihn mit Hilfe der Figur des «Terminators» aus den Schwarzenegger-Filmen festzunehmen und in eine erlebnispädagogische Maßnahme nach Kanada zu schicken. Die Kollegin erlebt durch die Imagination große emotionale Entlastung.

Zum Abschluss stellt die Supervisorin im Rollenspiel einen narzisstischen Patienten dar. Nina F. gelingt es hervorragend und ohne Angst, ihn in der von ihr zu Beginn des Stuhldialoges vorgeschlagenen Weise mit einem schwierigen Thema zu konfrontieren. Einige Wochen nach der Sitzung berichtet Nina F., dass es ihr problemlos gelungen sei, das Erarbeitete in ihrer Arbeit umzusetzen, darüber hinaus sei sie auch in ihrem Privatleben gegenüber übermäßig dominanten Kollegen oder Bekannten mittlerweile erstaunlich selbstbewusst.

\section{Diskussion}

In der Diskussion über Selbsterfahrung als Bestandteil der VT-Ausbildung [Rief et al., 2009] wird kritisch angemerkt, dass verhaltenstherapeutische Selbsterfahrung trivial werden kann, wenn Ausbildungskandidaten in der Selbsterfahrung verhaltenstherapeutische Methoden anwenden sollen, die eigentlich für die Behandlung schwerer Symptome entwickelt wurden. Dem gegenüber steht die Forderung, in die Selbsterfahrung gezielt stärker interpersonelles und emotionales Lernen zu integrieren und auf ausbildungsrelevante Inhalte zu fokussieren [Laireiter, 2009]. Dabei sollte beachtet werden, dass Konzepte, die gleichermaßen praxis- und personbezogene Ziele verfolgen, die breitesten positiven Effekte haben [Laireiter, 2000a].

In der Arbeit mit dem Modusmodell werden gerade interpersonelle und emotionale Aspekte konzeptgeleitet integriert und gleichermaßen praxis- und personbezogene Ziele verfolgt. Bezogen auf praxisorientierte Ziele zeigt sich dabei immer wieder, dass der Einsatz emotionaler Methoden in der Selbsterfahrung weitaus einfacher realisierbar ist als in der Patientenarbeit, da Therapeuten sehr gut in der Wahr- 
nehmung und Bearbeitung ihrer Emotionen geschult sind. Das heißt, Therapeuten stellen in der Regel sehr «leichte» Fälle da, die sich - z.B. im Rahmen von Kleingruppenarbeit in der Gruppenselbsterfahrung - gut zum Üben eigenen. Eine hohe Ausbildungsrelevanz ist dadurch gegeben, dass die Systematik des Einsatzes emotionaler Methoden in der Selbsterfahrung grundsätzlich übereinstimmt mit dem Vorgehen in der Therapie; dies wird jedoch nicht trivial, da keine schweren Symptome vorliegen müssen, um das Konzept sinnvoll einzusetzen.

Aktuell stellt diese Arbeit einen Vorschlag zur stärker konzeptgeleiteten und damit lehrbaren Integration emotionaler Methoden in die VT dar. Der Einsatz in Supervision und Selbsterfahrung erscheint dafür nach bisherigen Erfahrungen didaktisch besonders attraktiv. Mittel- und langfristig sollte jedoch empirisch geprüft werden, inwieweit die damit angestrebten Ziele erreicht werden können. Dabei ist kritisch in Betracht zu ziehen, dass es sich bei den vorgestellten Überlegungen um ein heuristisches Modell handelt und nicht um eine im engeren Sinne empirisch vollständig belegbare Theorie. Auch wenn erste Ansätze zur empirischen Überprüfung des Modells dessen Validität unterstützen [Arntz et al.,
2005; Lobbestael et al., 2008], sollten zukünftige Aktivitäten auch auf eine weitere Verbesserung der theoretischen Klarheit zielen.

Weiterhin ist es bei diesem Vorgehen in höchstem Maße notwendig, ethische Aspekte zu bedenken. Je mehr in die Biographie oder Persönlichkeit eines Ausbildungskandidaten eingegriffen wird, desto mehr stellt sich die Frage, wer den Kandidaten vor dem Selbsterfahrungsleiter schützt und wo die Grenzen erlaubter Interventionen sind. Selbstverständlich sind die Grundlagen ethischen Handelns zu beachten [Reimer, 2007]. Darüber hinaus ist es in jedem Falle zwingend notwendig, das Vorgehen mit dem Selbsterfahrungskandidaten abzusprechen und sein explizites Einverständnis einzuholen. Es sollte nicht mit diesem Konzept gearbeitet werden, wenn zwischen Selbsterfahrungsleiter und Ausbildungskandidat irgendeine Art der Abhängigkeit besteht.

\section{Disclosure Statement}

Es liegen keine finanziellen oder ideellen Interessenskonflikte vor.

\section{Literatur}

Arntz A, Klokman J, Sieswerda S: An experimental

test of the schema mode model of borderline personality disorder. J Behav Ther Exp Psychiatry 2005;36:226-239.

Arntz A, Tiesema M, Kindt M: Treatment of PTSD: A

comparison of imaginal exposure with and without imagery rescripting. J Behav Ther Exp Psychiatry 2007;38:345-370.

Arntz A: Schema therapy for cluster-C personality disorders. Keynote at the biannual meeting of the International Society of Schema Therapy. July 2010, Berlin, http://isst-online.com/node/157.

Bernstein D: Treating the untreatable: Schema therapy for psychopathic patients. Keynote at the biannual meeting of the International Society of Schema Therapy. July 2010, Berlin, http://isst-online.com/ node/157.

Farrell JM, Shaw IA, Webber MA: A schema-focused

approach to group psychotherapy for outpatients with borderline personality disorder: A randomized controlled trial. J Behav Ther Exp Psychiatry 2009; 40:317-328.

Frank R: Wohlbefinden von Psychotherapeutinnen und Psychotherapeuten: Evaluation von Selbsterfahrung während der Aus- und Weiterbildung; in Laireiter AR (Hrsg): Selbsterfahrung in Psychotherapie und Verhaltenstherapie. Empirische Befunde. Tübingen, dgvt-Verlag, 2000, pp 539-571.

Giesen-Bloo J, van Dyck R, Spinhoven P, van Tilburg

W, Dirksen C, van Asselt T, Kremers I, Nadort M, Arntz A: Outpatient psychotherapy for borderline personality disorder: Randomized clinical trial of schema-focused therapy vs transference-focused psychotherapy. Arch Gen Psychiatry 2006;63:649-658.
Holmes E, Arntz A, Smucker M: Imagery rescripting

in cognitive behaviour therapy: Images, treatment techniques and outcomes. J Behav Ther Exp Psychiatry 2007:38:297-305.

Jacob GA, Arntz A: Schematherapie in der Praxis. Weinheim, Beltz, 2011.

Jacob GA, Lieb K, Berger M: Schwierige Gesprächssituationen in Psychiatrie und Psychotherapie. München, Elsevier, 2009.

Laireiter AR: Selbsterfahrung in der Verhaltenstherapie: Konzepte und empirische Befunde; in Laireiter AR (Hrsg): Selbsterfahrung in Psychotherapie und Verhaltenstherapie. Empirische Befunde. Tübingen, dgvt-Verlag, 2000a, pp 365-412.

Laireiter AR (Hrsg): Selbsterfahrung in Psychotherapie und Verhaltenstherapie. Empirische Befunde. Tübingen, dgvt-Verlag, 2000b.

Laireiter AR: Wenn wir über Selbsterfahrung reden, dann müssen wir über Ausbildung reden! Verhaltenstherapie 2009;19:191-193.

Lieb H (Hrsg): Selbsterfahrung für Psychotherapeuten: Konzepte, Praxis, Forschung. Göttingen, Hogrefe, 1998.

Lobbestael J, van Vreeswijk MF, Arntz A: An empirical test of schema mode conceptualizations in personality disorders. Behav Res Ther 2008;46:854-860.

Lobbestael J, Arntz A, Bernstein DP: Disentangling

the relationship between different types of childhood maltreatment and personality disorders. J Pers Disord 2010;24:285-295.
Nadort M, Arntz A, Smit JH, Giesen-Bloo J, Eikelenboom M, Spinhoven P, van Asselt T, Wensing M van Dyck R: Implementation of outpatient schema therapy for borderline personality disorder with versus without crisis support by the therapist outside office hours: A randomized trial. Behav Res Ther 2009;47:961-973.

Reimer C: Ethische Aspekte der Psychotherapie; in Reimer C, Eckert J, Hautzinger M, Wilke E (Hrsg): Psychotherapie. Ein Lehrbuch für Ärzte und Psychologen. Heidelberg, Springer, 2007, pp 745-759.

Rief W, Freyberger HJ, Sartory G: Macht Selbster-

fahrung bessere Psychotherapeuten? Verhaltenstherapie 2009;19:53-55.

Roediger E, Jacob G (Hrsg): Fortschritte der Schematherapie. Göttingen, Hogrefe, 2010.

Weertman A, Arntz A: Effectiveness of treatment of

childhood memories in cognitive therapy for personality disorders: A controlled study contrasting methods focusing on the present and methods focusing on childhood memories. Behav Res Ther 2007;45:2133-2143.

Young JE, Klosko S, Weishaar ME: Schematherapie. Ein praxisorientiertes Handbuch. Paderborn, Junfermann, 2005. 